\title{
Lipopolysaccharide-binding protein is a negative regulator of adipose tissue browning in mice and humans
}

\author{
Aleix Gavaldà-Navarro ${ }^{1,2,3,4}$ - José M. Moreno-Navarrete ${ }^{4,5}$. \\ Tania Quesada-López ${ }^{1,2,3,4}$ - Montserrat Cairó ${ }^{1,2,3,4}$ - Marta Giralt Mar, $^{1,2,4}$. \\ José M. Fernández-Real ${ }^{4,5}$ - Francesc Villarroya ${ }^{1,2,3,4}$
}

Received: 5 January 2016 / Accepted: 25 May 2016/Published online: 25 June 2016

(C) Springer-Verlag Berlin Heidelberg 2016

\begin{abstract}
Aims/hypothesis Adipocyte lipopolysaccharide-binding protein (LBP) biosynthesis is associated with obesity-induced adipose tissue dysfunction. Our purpose was to study the role of LBP in regulating the browning of adipose tissue.

Methods Adult mice were maintained at $4^{\circ} \mathrm{C}$ for 3 weeks or treated with the $\beta_{3}$-adrenergic agonist, CL316,243, for 1 week to induce the browning of white fat. Precursor cells from brown and white adipose tissues were cultured under differentiation-inducing conditions to yield brown and beige/ brite adipocytes, respectively. In vitro, $L b p$ was knocked down in 3T3-L1 adipocytes, and cells were treated with recombinant LBP or co-cultured in transwells with control 3T3-L1 adipocytes. Wild-type and $L b p$-null mice, fed a standard or high fat diet (HFD) for 15 weeks, were also used in investigations. In humans, subcutaneous and visceral adipose
\end{abstract}

Electronic supplementary material The online version of this article (doi:10.1007/s00125-016-4028-y) contains peer-reviewed but unedited supplementary material, which is available to authorised users.

Francesc Villarroya

fvillarroya@ub.edu

1 Department of Biochemistry and Molecular Biology, University of Barcelona, Av. Diagonal 643, 08028 Barcelona, Catalonia, Spain

2 The Institute of Biomedicine of the University of Barcelona (IBUB), Barcelona, Spain

3 Institut de Recerca Pediàtrica Hospital Sant Joan de Déu, Barcelona, Spain

4 Centro de Investigación Biomédica en Red de Fisiopatología de la Obesidad y Nutrición (CIBEROBN), Spain http://www.ciberobn.es/en

5 Section of Diabetes, Endocrinology and Nutrition, Institut d'Investigació Biomèdica de Girona (IdIBGi), Hospital of Girona "Dr Josep Trueta", Girona, Spain tissue samples were obtained from a cohort of morbidly obese participants.

Results The induction of white fat browning by exposure of mice to cold or CL316,243 treatment was strongly associated with decreased $L b p$ mRNA expression in white adipose tissue. The acquisition of the beige/brite phenotype in cultured cells was associated with downregulation of $L b p$. Moreover, silencing of $L b p$ induced the expression of brown fat-related genes in adipocytes, whereas LBP treatment reversed this effect. $L b p$-null mice exhibited the spontaneous induction of subcutaneous adipose tissue browning, as evidenced by a remarkable increase in Ucp1 and Dio2 gene expression and the appearance of multivacuolar adipocyte clusters. The amount of brown adipose tissue, and brown adipose tissue activity were also increased in $L b p$-null mice. These changes were associated with decreased weight gain in $L b p$-null mice and protection against HFD-induced inflammatory responses, as shown by reduced IL-6 levels. However, rather than improving glucose homeostasis, these effects led to glucose intolerance and insulin resistance.

Conclusions/interpretation LBP is identified as a negative regulator of the browning process, which is likely to contribute to the obesity-promoting action of LBP. The deleterious metabolic effects of LBP deletion are compatible with the concept that the appropriate regulation of inflammatory pathways is necessary for a healthy systemic metabolic profile, regardless of body weight regulation.

Keywords Brown adipose tissue $\cdot$ Browning $\cdot$ High-fat diet . Lipopolysaccharide-binding protein $\cdot$ Obesity
Abbreviations
BAT Brown adipose tissue
eWAT Epididymal white adipose tissue
HFD High fat diet 


$\begin{array}{ll}\text { iBAT } & \text { Interscapular brown adipose tissue } \\ \text { ITT } & \text { Insulin tolerance test } \\ \text { iWAT } & \text { Inguinal white adipose tissue } \\ \text { LBP } & \text { Lipopolysaccharide-binding protein } \\ \text { LPS } & \text { Lipopolysaccharide } \\ \text { SAT } & \text { Subcutaneous adipose tissue } \\ \text { shControl } & \text { Control small hairpin RNA } \\ \text { shLBP } & \text { Small hairpin RNA-mediated LBP knockdown } \\ \text { shRNA } & \text { Small hairpin RNA } \\ \text { UCP1 } & \text { Uncoupling protein 1 } \\ \text { VAT } & \text { Visceral adipose tissue } \\ \text { WAT } & \text { White adipose tissue } \\ \text { WT } & \text { Wild-type }\end{array}$

\section{Introduction}

Obesity is characterised by lipid accumulation in white adipose tissue (WAT) as a consequence of a disturbed energy balance due to increased food energy intake and/or lowered energy expenditure. Importantly, it is associated with an increased incidence of metabolic disorders, especially type 2 diabetes mellitus [1].

Brown adipose tissue (BAT) is characterised by the presence of multivacuolar adipocytes with a thermogenic capacity that reflects the natural uncoupling of their mitochondria, as mediated by uncoupling protein 1 (UCP1). BAT is the main site of adaptive energy expenditure in response to cold and possibly to diet [2]. In experimental models, enhanced BAT activity is associated with protection against obesity, whereas impaired BAT activity favours an obese phenotype. Moreover, BAT is a major site of lipid breakdown and glucose uptake; therefore, BAT activation is associated with improvements in hyperlipidaemia and hyperglycaemia [3, 4]. In humans, it was previously thought that BAT is only present in neonates. However, more recent research has established that adult humans retain substantial BAT activity [5, 6]. In addition to the presence of BAT at defined anatomical locations, recent studies have attached special relevance to the WAT browning process [7]. This phenomenon consists of the appearance of functionally thermogenic brown adipocyte-like cells (so-called beige or brite adipocytes) in WAT depots. Several studies in mice have indicated that the capacity to activate this process may protect against diet-induced obesity and systemic metabolic disturbances [8,9], although the actual relevance of adipose tissue browning to metabolism is still a matter of debate $[10,11]$.

Lipopolysaccharide (LPS)-binding protein (LBP) is a type I acute-phase reactant protein, thought to be mainly produced by the liver [12]. LBP facilitates the binding of the lipid A component of LPS to CD14 and toll-like receptor 4 (TLR4) [13], thus modulating the immunostimulatory capacity of LPS in bacterial infections [14]. LBP is also present in adipose tissues and high levels of LBP synthesis in white adipocytes are associated with WAT dysfunction in obesity [15]. LBP expression is associated with inflammatory markers and is increased with metabolic deterioration and insulin resistance in obese patients [16]. Here, we sought to establish the role of LBP in the browning of WAT. We investigated the association of $L b p$ expression with browning and studied alterations in browning and metabolism in $L b p$-null mice.

\section{Methods}

Exposure of mice to chronic cold and treatment with CL316,243 The care and use of mice was carried out in accordance with the European Community Council Directive 86/609/EEC and approved by the Institutional Animal Care Committee of the University of Barcelona. C57BL/6 mice (Harlan Laboratories; Indianapolis, IN, USA) were maintained under standard conditions $(12 \mathrm{~h}$ light/12 h dark cycle, $21 \pm 1^{\circ} \mathrm{C}$ ). Where indicated, 3-month-old mice were exposed to $4^{\circ} \mathrm{C}$ for 3 weeks, or 2-month-old mice received daily i.p injections with $1 \mathrm{mg} / \mathrm{kg}$ CL316,243 for 8 days (see electronic supplementary material [ESM] Methods; exposure of mice to chronic cold, and treatment of mice with CL316,243, for further details).

Adipocyte culture For primary adipocyte cultures, the stromal-vascular fraction was isolated from the inguinal WAT (iWAT) and interscapular BAT (BAT) depots of 1month-old mice. Pre-adipocytes were induced to differentiate into beige/brite and brown adipocytes, respectively, as previously reported $[17,18]$. Differentiated beige/brite and brown adipocytes were treated with $0.5 \mu \mathrm{mol} / \mathrm{l}$ noradrenaline (norepinephrine) for $6 \mathrm{~h}$. Differentiation of 3T3-L1 fibroblasts (ATCC; LGC Standards, Barcelona, Spain) was induced by treatment with $0.86 \mu \mathrm{mol} / \mathrm{l}$ insulin, $0.5 \mu \mathrm{mol} / \mathrm{l}$ dexamethasone and $0.5 \mathrm{mmol} / 13$-isobutyl-1-methylxanthine (IBMX) for 2 days, followed by $0.86 \mu \mathrm{mol} / 1$ insulin alone for 5 days. See ESM Methods for further details.

LBP knockdown and co-culture experiments Stable silencing of $L b p$ was achieved by transfecting 3T3-L1 fibroblasts with small hairpin (interfering) RNA (shRNA) targeting mouse Lbp (shLBP)or control shRNA (shControl) (Sigma Mission shRNA; Sigma-Aldrich, St Louis, MO, USA) as previously reported [16], generating transfected cells described in this paper as shRNA-mediated LBP knockdown (shLBP) or control (shControl) cells, respectively. Where indicated, shLBP adipocytes were incubated with $10 \mathrm{ng} / \mathrm{ml}$ LBP (R\&D Systems; Minneapolis, MN, USA) during differentiation. Differentiated shLBP or shControl 3T3-L1 adipocytes were placed on one side of a Transwell plate (VWR- 
International Eurolab, Barcelona, Spain) and co-cultured with shLBP adipocytes on the other side. See ESM Methods for further details.

Lbp-null mouse studies $L b p$-knockout mice (C.129P2Lbptm1Jack/J, Balb strain background; obtained from The Jackson Laboratory, Bar Harbor, ME, USA) were maintained at $21 \pm 1^{\circ} \mathrm{C}$. Five-week-old male $L b p$-null and wild-type (WT) littermate mice were fed standard chow (CTRL) or a high fat diet (HFD) for 15 weeks. For GTTs, $2.5 \mathrm{~g}$ glucose/kg was administered i.p. to mice starved for $6 \mathrm{~h}$. For insulin tolerance tests (ITTs), $0.75 \mathrm{IU} / \mathrm{kg}$ insulin (Actrapid; Novo Nordisk, Bagsvaerd, Denmark) was administered i.p. Plasma glucose (Accutrend; Roche Diagnostics, Mannheim, Germany), plasma insulin (BioVendor, Brno, Czech Republic); adiponectin (Life Technologies, Foster City, CA, USA); leptin, resistin and IL-6 (Millipore; Billerica, MA, USA); NEFA and 3-hydroxybutyrate (Wako Chemicals; Neuss, Germany); and triacylglycerols (Sigma-Aldrich) were assessed. Samples of iBAT or iWAT (25-50 mg) were incubated with $55.5 \mathrm{kBq} / \mathrm{ml}\left[\mathrm{U}^{14}{ }^{14} \mathrm{C}\right] \mathrm{D}$-glucose (Hartmann Analytic, Braunschweig, Germany) for $3 \mathrm{~h}$. Disintegrations per minute (DPM) from ${ }^{14} \mathrm{CO}_{2}$ retained in a $\mathrm{CO}_{2}$ trap and $\left[{ }^{14} \mathrm{C}\right]$ tissue lipid extracts [19] were measured (Packard Instrument Company, Meriden, CT, USA). See ESM Methods for further details.

Human study Paired subcutaneous and visceral adipose tissue (VAT) samples $(n=38)$ from a cohort of morbidly obese $\left(\right.$ BMI $\left.>35 \mathrm{~kg} / \mathrm{m}^{2}\right)$ participants were studied. These participants were recruited, gave written informed consent, and were validated and approved by the ethical committee at the Hospital of Girona 'Dr Josep Trueta'. Samples were obtained from subcutaneous adipose tissue (SAT) and VAT depots during elective surgical procedures. See ESM Methods for further details.

RNA isolation, cDNA synthesis and real-time PCR Total RNA was isolated (Macherey-Nagel, Düren, Germany) from BAT, iWAT, eWAT and liver samples from mice, and SAT and VAT samples from humans and retrotranscribed using TaqMan Reverse Transcription Reagents (Life Technologies). For the quantitative analysis of mRNA, TaqMan quantitative real-time PCR was performed on a 7500 Real-Time PCR System (Life Technologies) using the specific primer pair/probe sets, as described in ESM Table 1. See ESM Methods for further details.

Western blot assays Western blot analysis was performed on BAT and iWAT samples following standard procedures, using primary anti-UCP1 (1:1000; Abcam, Cambridge, UK) and anti- $\beta$-actin (1:5000; Sigma-Aldrich) antibodies. Both antibodies were diluted in $1 \times$ PBS containing $0.1 \%$
Tween-20, as per manufacturer's instructions. See ESM Methods for further details.

Statistical methods Results are expressed as mean \pm SEM. Statistical analyses were performed using GraphPad Prism 6 (La Jolla, CA, USA). The statistical significance of differences was assessed using unpaired Student's $t$ tests, one-way ANOVA with Tukey's multiple comparison test, or two-way ANOVA with Bonferroni post-testing, as appropriate.

\section{Results}

Induction of browning in subcutaneous WAT is associated with reduced $\boldsymbol{L} \boldsymbol{b} \boldsymbol{p}$ expression We subjected mice to longterm cold exposure $\left(4^{\circ} \mathrm{C}\right.$ for 3 weeks $)$ and then examined $L b p$ gene expression in adipose tissue depots. As expected, cold exposure increased expression of the thermogenesisrelated genes Ucp 1, Sirt3, Fgf21 and Ppargcla in subcutaneous WAT (i.e. iWAT; Fig. 1d-h); it also induced the browning process, indicated by the appearance of large amounts of multilocular adipocytes (Fig. 1a,b). These changes were associated with a marked reduction in $L b p$ expression (Fig. 1c). $L b p$ expression in visceral WAT (i.e. epididymal WAT [eWAT]), a WAT site less prone to browning, was also decreased in mice exposed to chronic cold, albeit to lesser extent (ESM Fig. 1a). In contrast, chronic cold exposure did not modify $L b p$ expression in iBAT (ESM Fig. 1h), even though we observed significant induction of thermogenesisrelated genes in this tissue (ESM Fig. 1i-n).

Chronic treatment of mice with the $\beta_{3}$-adrenergic agonist CL316,243 (a model of induction of browning in iWAT and eWAT [20]), triggered the appearance of multilocular adipocytes (Fig. 11, m), induced thermogenesis-related gene expression (Fig. 1o-v) and strongly repressed $L b p$ gene expression (Fig. 1n) in iWAT and eWAT (ESM Fig. 2a-i). Although iBAT also showed signs of activation following CL316,243 treatment (e.g. the induction of Dio2 and $B m p 8 b), L b p$ expression was not significantly altered in this tissue (ESM Fig. 21-r). Overall, our results suggest that the downregulation of LBP is more strongly associated with WAT browning than with the induction of thermogenesis in iBAT.

$L b p$ expression is involved in the repression of browning in adipocytes In order to determine whether the inverse relationship between $L b p$ expression and browning is a cellautonomous phenomenon, we used rosiglitazone-induced browning (i.e. acquisition of the beige/brite phenotype) in precursor cells obtained from iWAT $[17,21]$. Incubation of iWAT precursor cells with rosiglitazone during differentiation induced the expression of several marker genes for browning (Fig. 2b-f), and repressed $L b p$ expression (Fig. 2a), without 
Fig. 1 Cold and $\beta_{3}$-adrenergic induction of iWAT browning is associated with reduced $L b p$ expression. (a, b) Micrographs (magnification $\times 20$ ) of iWAT from (a) control mice or (b) mice maintained at $4{ }^{\circ} \mathrm{C}$ for 3 weeks (chronic cold). (c--k) Transcript levels of (c) $L b p$, (d) $U c p 1$,
(e) $\operatorname{Prdm16}$, (f) Fgf21,
(g) Ppargcla, (h) Sirt3,
(i) Slc2a4, (j) Pparg and

(k) Adipoq from control mice (white bars) or mice maintained at $4^{\circ} \mathrm{C}$ for 3 weeks (black bars).

$(\mathbf{l}, \mathbf{m})$ Micrographs $($ magnification $\times 20)$ of iWAT from (I) control mice or (m) mice injected daily with $1 \mathrm{mg} / \mathrm{kg}$ CL316,243 for 8 days.

(n-v) Transcript levels of (n) $L b p$, (o) Ucp1, (p) Prdm16, (q) Fgf21, (r) Ppargcla, (s) Sirt3, (t) Slc2a4, (u) Pparg and (v) Adipoq from control mice (white bars) or mice injected with CL316,243 (black bars). mRNA levels are normalised to $18 \mathrm{~S}$ rRNA levels.

Data are presented as mean

\pm SEM of six independent samples per group. $* p<0.05$,

$* * p<0.01$, and $* * * p<0.001$ a
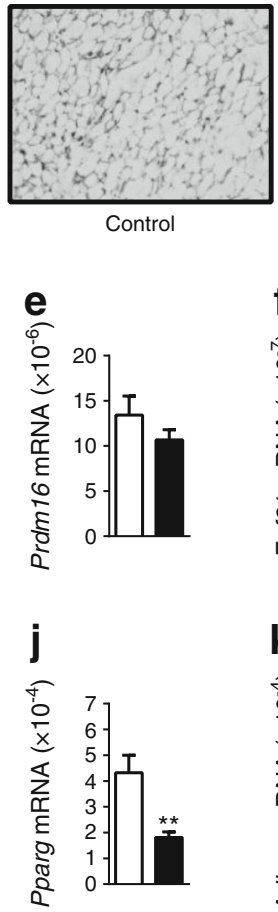

n
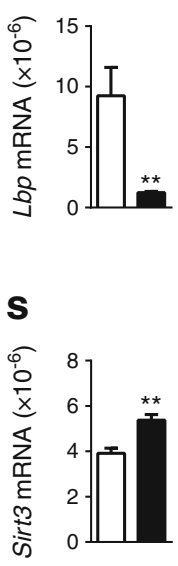

b

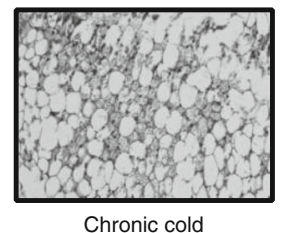

f
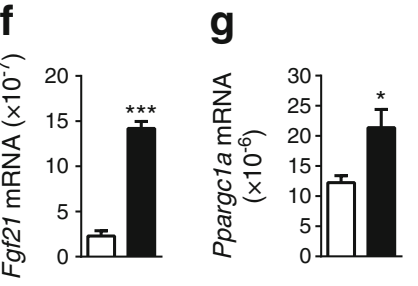

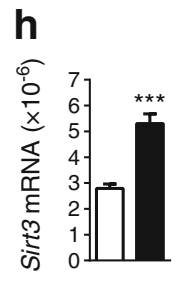
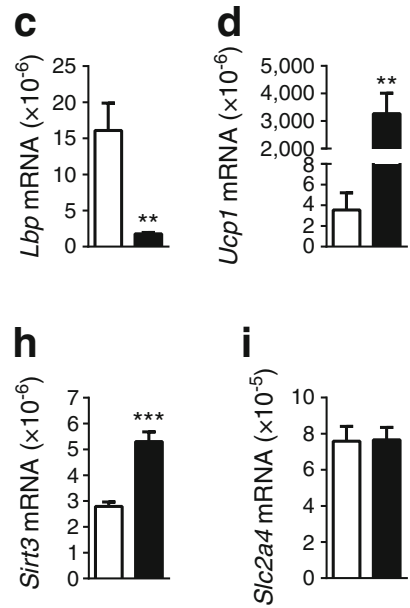

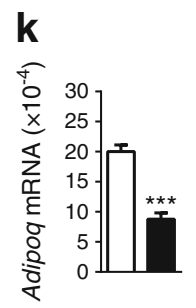

I

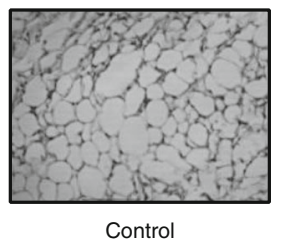

m

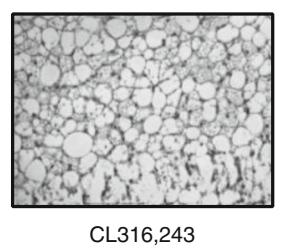

p
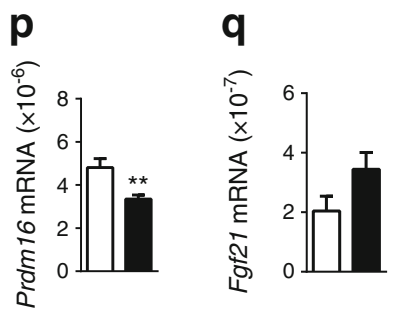

r
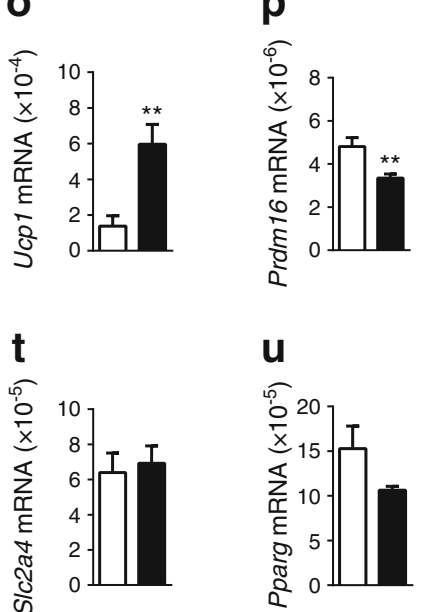

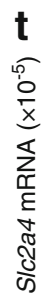

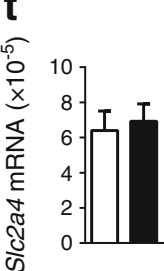

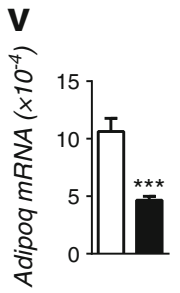

affecting the expression of the general adipogenesis-related genes Slc2a4 (also known as Glut4), Adiponectin (Adipoq) and Pparg (Fig. 2g-i). These data confirm that negative regulation of LBP expression is associated with the adipocyte browning. Moreover, treatment of differentiated beige/brite adipocytes with noradrenaline repressed $L b p$ and induced Ucpl gene expression (ESM Fig. 3a,b); however, noradrenaline did not modify $L b p$ expression in brown adipocytes (ESM Fig. 3c).

To investigate whether LBP has a direct role in the browning process, we studied the effects of shRNAmediated LBP knockdown in 3T3-L1 adipocytes. After 7 days of differentiation, shLBP exhibited increased mRNA levels of several thermogenesis-related genes, including Ucp 1,
Ppargcla and Sirt3 (Fig. 2k-o). Treatment of shLBP cells with recombinant LBP rescued the induction of Ucp 1 mRNA expression induced by loss of $L b p$ (Fig. 2s). Moreover, shLBP adipocytes subjected to transwell co-culture with control adipocytes showed lower levels of Ucp 1 mRNA expression compared with those co-cultured with shLBP cells (Fig. 2t). Overall, these data suggest that the downregulation of $L b p$ represses browning in a cell-autonomous manner.

Lbp-null mice exhibit reduced body weight gain accompanied by an induction of the browning process in iWAT To study the role of LBP in vivo, $L b p$-null mice and WT littermate controls were fed standard chow (CTRL) or an 

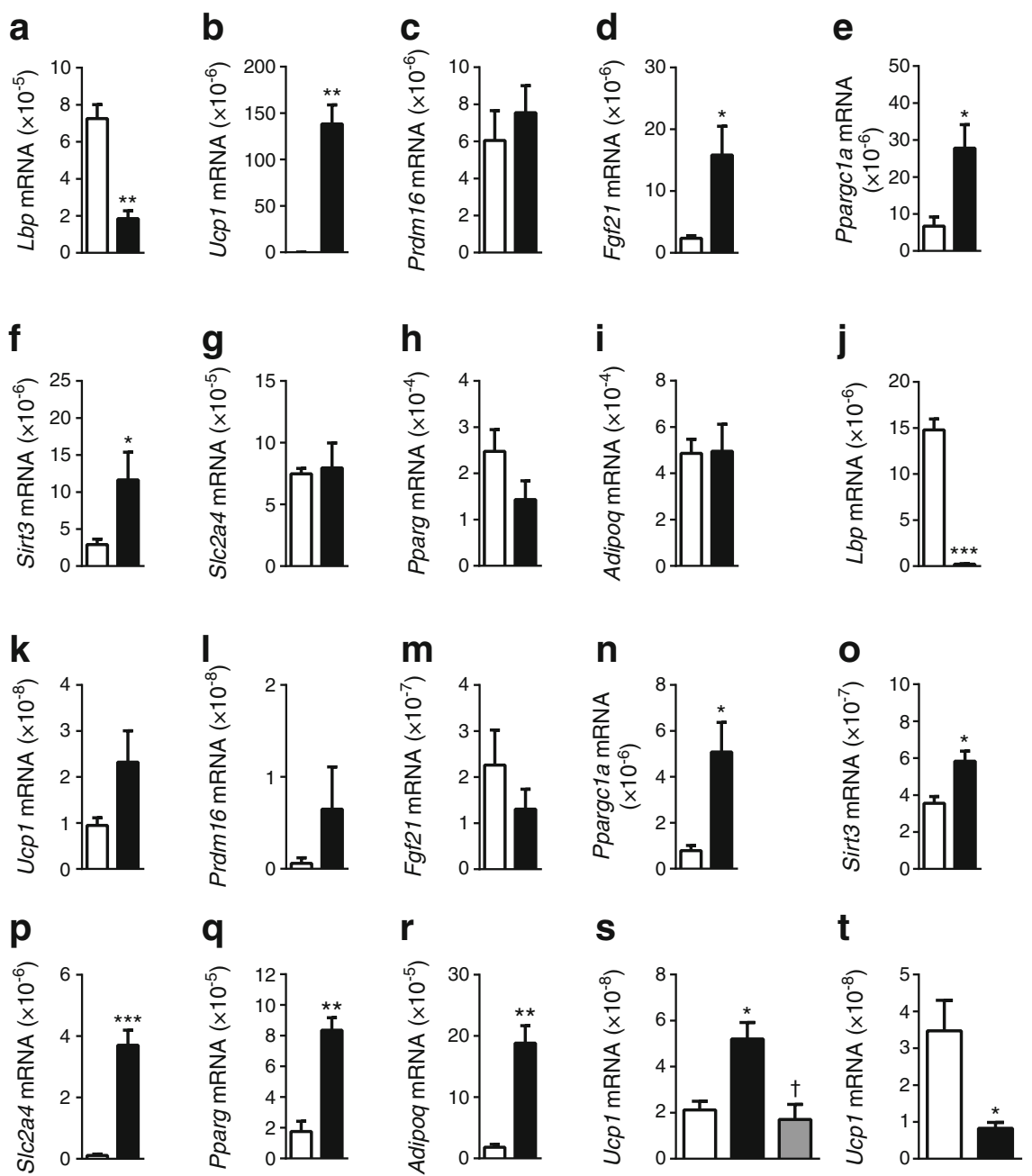

Fig. $2 L b p$ and the expression of browning-related genes are inversely regulated in a cell-autonomous manner. (a-i) Transcript levels of (a) $L b p$, (b) Ucp1, (c) Prdm16, (d) Fgf21, (e) Ppargcla, (f) Sirt3, (g) Slc2a4, (h) Pparg and (i) Adipoq, in WAT-derived primary white adipocytes (white bars) or rosiglitazone-induced beige/brite adipocytes (black bars). (j-r) Transcript levels of (j) Lbp, (k) Ucp1, (I) Prdm16, (m) Fgf21, (n) Ppargcla, (o) Sirt3, (p) Slc2a4, (q) Pparg and (r) Adipoq in 3T3-L1 adipocytes transfected with a control shRNA (shControl; white bars) or an shRNA targeting LBP (black bars). (s) Transcript levels of

Ucp1 in control 3T3-L1 adipocytes (white bars) or LBP-knockdown adipocytes untreated (black bar) or treated (grey bar) with $10 \mathrm{ng} / \mathrm{ml}$ LBP. (t) Transcript levels of Ucpl in LBP-knockdown 3T3-L1 adipocytes co-cultured with LBP-knockdown (white bar) or control 3T3-L1 adipocytes (black bar). mRNA levels are normalised to 18S rRNA levels. Data are presented as mean \pm SEM of 4-7 independent samples per group. ${ }^{*} p<0.05, * * p<0.01$ and $* * * p<0.001$ vs control ${ }^{\dagger} p<0.05$ for treated vs non-treated LBP-knockdown)

HFD for 15 weeks. At the start of the experiment (5-week-old mice), body weight was similar between WT and $L b p$-null mice. The body weight of WT mice increased to $144 \%$ of the initial weight under standard feeding conditions and to $162 \%$ when fed an HFD (Fig. 3a,b). When fed a standard (CTRL) diet, the increase in body weight was significantly lower in Lbp-null mice (135\%) than in WT mice on the same diet. On an HFD, the body weight of $L b p$-null mice increased to only $145 \%$ of their initial weight; this increase was not significantly different from the body weight increase in $L b p$-null mice on a standard diet. $L b p$-null mice did not show significant changes in food intake relative to WT mice under standard diet $(105 \pm 4 \% \mathrm{~kJ} /$ day in $L b p$-null vs WT) or HFD $(112 \pm 8 \% \mathrm{~kJ} /$ day in $L b p$-null vs $\mathrm{WT})$ conditions. Oxygen consumption was not significantly different among the different experimental groups (Fig. 3c).

The RQ was significantly decreased in WT mice fed HFD (Fig. 3d). In $L b p$-null mice, RQ did not change significantly in response to an HFD, indicating a somewhat altered metabolic flexibility to adapt to HFD as a consequence of $L b p$ invalidation.

Lbp-null mice showed no statistically significant alterations in blood glucose levels (Fig. 3e), whereas Lbp-null mice had higher plasma triacyclglycerol levels when maintained on an HFD diet (Fig. 3f). Insulinaemia was strongly increased in $L b p$-null mice relative to WT mice on an HFD (Fig. $3 \mathrm{~g}$ ). There were no differences in plasma NEFA, 3-hydroxybutyrate, leptin, adiponectin or resistin levels between WT and $L b p$-null 
Fig. 3 Lbp-null mice have diminished body weight gain, with impaired glucose homeostasis. (a) Time course of mouse body weight, (b) final body weight gain, (c) oxygen consumption and (d) RQ.

(e-h) Plasma levels of

(e) glucose, (f) triacylglycerols, (g) insulin and (h) IL-6. White bars, WT mice; black bars, Lbp-null mice. (i) Glucose tolerance and (j) insulin tolerance tests in WT and Lbp-null mice after 15 weeks on a standard diet (CTRL) or HFD. White circles, WT mice, standard diet; White squares, WT mice, HFD; black circles, $L b p$-null mice, standard diet; black squares, $L b p$-null mice, HFD. Data are presented as mean \pm SEM of 6-7 mice per group. ${ }^{*} p<0.05, * * p<0.01$ and $* * * p<0.001$, WT vs LBP-null mice; ${ }^{\dagger} p<0.05,{ }^{\dagger \dagger} p<0.01$ and ${ }^{\dagger \dagger} p<0.001$, CTRL vs HFD
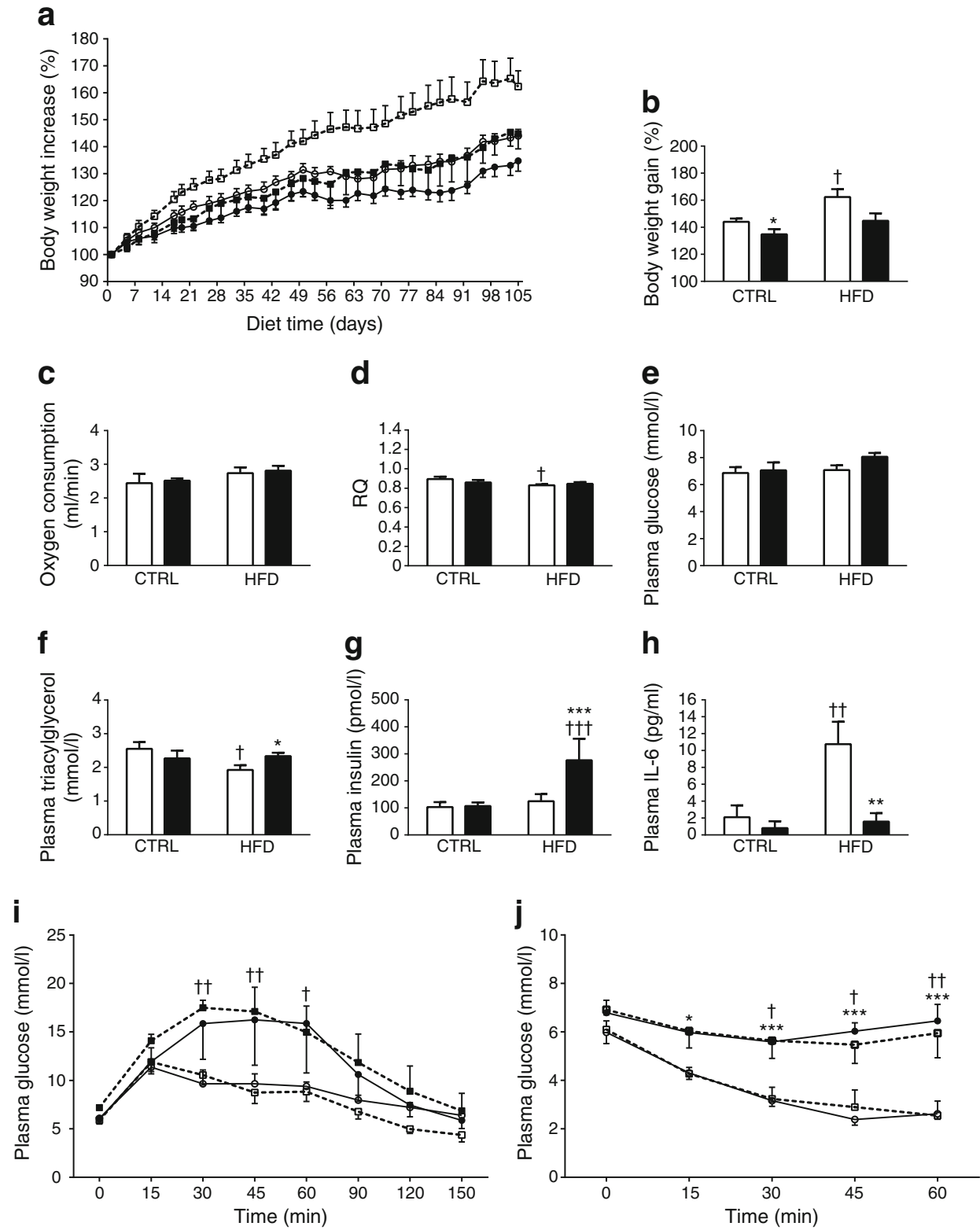

mice under CTRL or HFD conditions (ESM Table 2). Of the proinflammatory factors, HFD strongly induced circulating IL-6 levels in WT mice; this effect was totally ablated in Lbp-null mice (Fig. 3h). Consistent with the Balb genetic background of these mice, HFD did not affect glucose tolerance or the insulin response of WT mice, as determined by GTTs and ITTs (Fig. 3i,j) [22]. However, Lbp-null mice exhibited marked glucose intolerance and reduced insulin sensitivity on both a standard diet and HFD.

Under both standard diet and HFD conditions, Lbp-null mice showed a marked induction of the browning phenotype markers Ucp1 and Dio2 in iWAT (Fig. 4a,b). Other marker genes, including Prdm16, Fgf21 and Ppargcla (Fig. 4c-e), showed a similar trend that did not reach statistical significance between groups, although multifactorial
ANOVA analysis revealed a significant increase in $F g f 21$ ( $p=0.03$ ) for the $L b p$-null factor. iWAT depots showed clusters of multivacuolar adipocytes (with beige/brite morphology) in Lbp-null mice, consistent with the gene expression data (Fig. $4 \mathrm{j}-\mathrm{m}$ ). Expression of genes involved in overall adipogenesis (Pparg, Leptin [Lep], and Adipoq) did not change (Fig. $4 \mathrm{~g}-\mathrm{i}$ ), whereas multifactorial ANOVA analysis revealed a significant increase in Slc2a4 $(p=0.04)$ for the $L b p$-null factor. The amount of UCP1 protein per iWAT depot was higher in $L b p$-null mice than in WT mice under both standard diet and HFD conditions (Fig 4p). Under conditions that promoted the highest browning, the amount of UCP1 protein in iWAT was around $8 \%$ of the levels observed in iBAT. Explants of iWAT from $L b p$-null mice had an increased rate of glucose oxidation (Fig. $4 \mathrm{q}$ ), but no change 
Fig. 4 Lbp-null mice exhibit induction of iWAT browning. $(\mathbf{a}-\mathbf{i}, \mathbf{n}, \mathbf{o})$ Transcript levels of (a) Ucp1, (b) Dio2, (c) Prdm16, (d) Fgf21, (e) Ppargcla,

(f) Slc2a4, (g) Pparg, (h) Lep, (i) Adipoq, (n) Il6 and (o) Tnfa in iWAT from WT (white bars) and Lbp-null (black bars) mice fed a standard diet (CTRL) or an HFD for 15 weeks. (j-m) Micrographs (magnification $\times 20$ ) of iWAT sections. (p) UCP1 protein per iWAT depot. (q) Glucose oxidation and (r) glucose incorporation into lipids in iWAT explants from WT (white bars) and $L b p$-null (black bars) mice. mRNA levels are normalised to 18S rRNA levels. Data are presented as mean \pm SEM of 4-6 independent samples per group. ${ }^{*} p<0.05,{ }^{*} p<0.01$ and $* * * p<0.001$, WT vs $L b p$-null mice; ${ }^{\dagger} p<0.05$, CTRL vs HFD. DPM, disintegrations per minute a
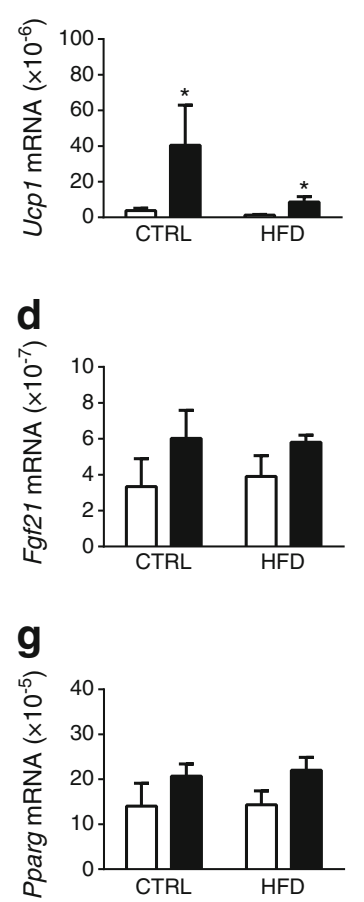

b

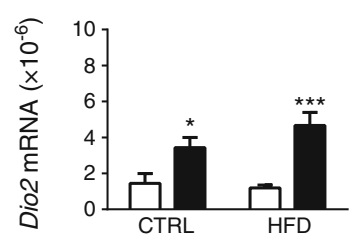

e

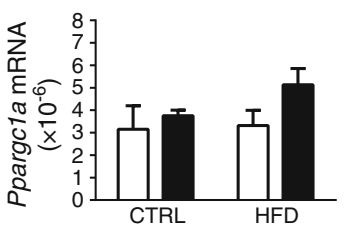

h

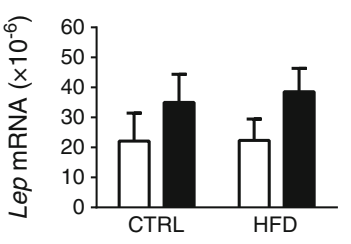

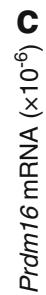
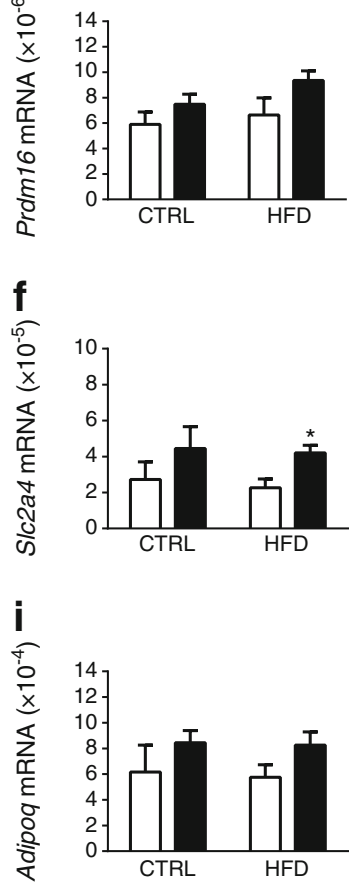

m

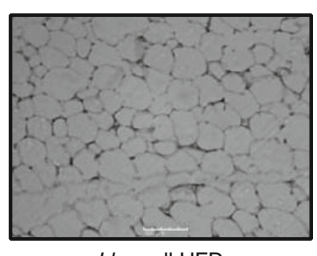

Lbp-null HFD

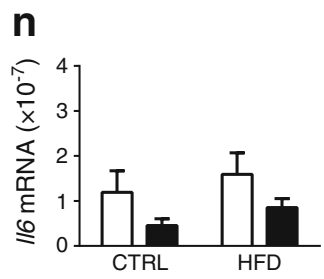

I

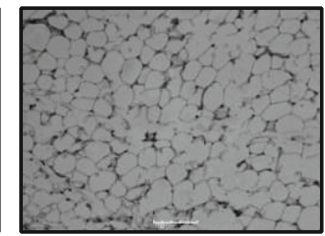

WT HFD
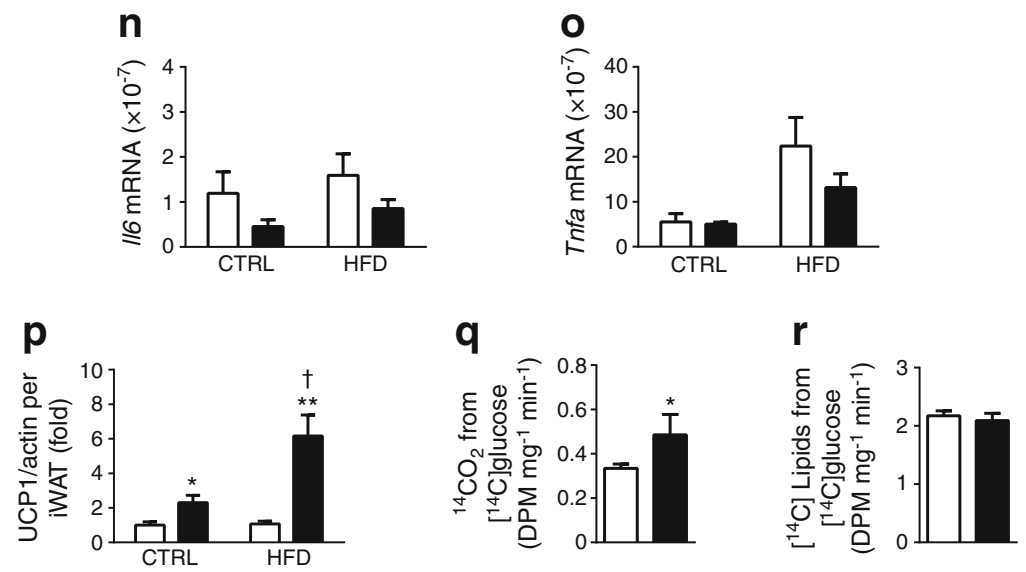

in the incorporation of glucose into lipids (Fig. 4r). This result indicates that oxidative pathways are enhanced in iWAT from Lbp-null mice, which is consistent with the molecular and morphological signs of browning in iWAT.

No evidence of significant browning due to $L b p$ gene ablation was detected in eWAT under any feeding condition, whether determined by gene expression analysis (with the exception of Dio2; ESM Fig. 4) or microscopic examination (data not shown).

iBAT in $\boldsymbol{L} \boldsymbol{b} \boldsymbol{p}$-null mice iBAT weight tended to be higher in Lbp-null mice relative to controls (Fig. 5a) and total iBAT protein content was significantly higher in $L b p$-null mice (Fig. 5b), indicating functional hypertrophy of iBAT in 
Fig. 5 iBAT in $L b p$-null mice. (a) iBAT weight, (b) iBAT protein content and (c) surface temperature at the iBAT site in WT (white bars) and $L b p$-null (black bars) mice fed a standard $\operatorname{diet}$ (CTRL) or an HFD. (d) Ucp I transcript levels and (e) UCP1 protein content per iBAT depot. (f) Representative immunoblot showing UCP1 protein levels in iBAT (40 $\mu \mathrm{g}$ protein/lane).

(g-k) Transcript levels of

(g) Dio2, (h) Prdm16, (i) Bmp8b,

(j) Ppargcla and (k) Fgf21 in

iBAT. mRNA levels are normalised to $18 \mathrm{~S}$ rRNA levels.

Data are presented as mean

\pm SEM of 4-6 independent samples per group. ${ }^{*} p<0.05$ and $*_{*}^{*} p<0.01$, WT vs $L b p$-null mice; ${ }^{\dagger} p<0.05$, CTRL vs HFD
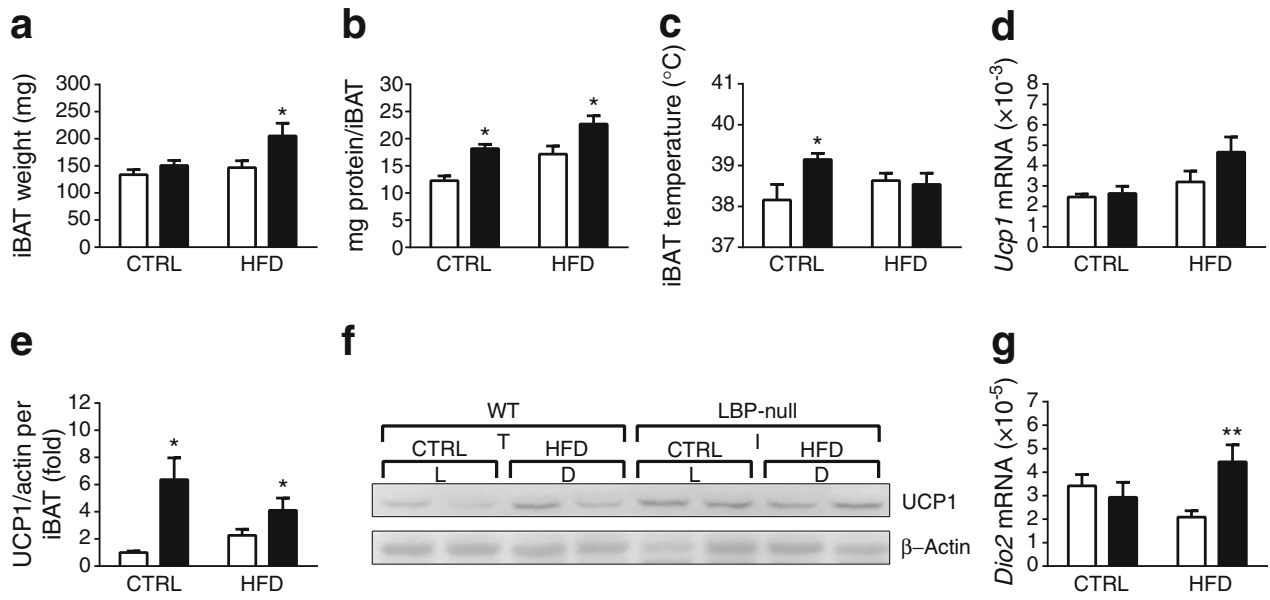

f
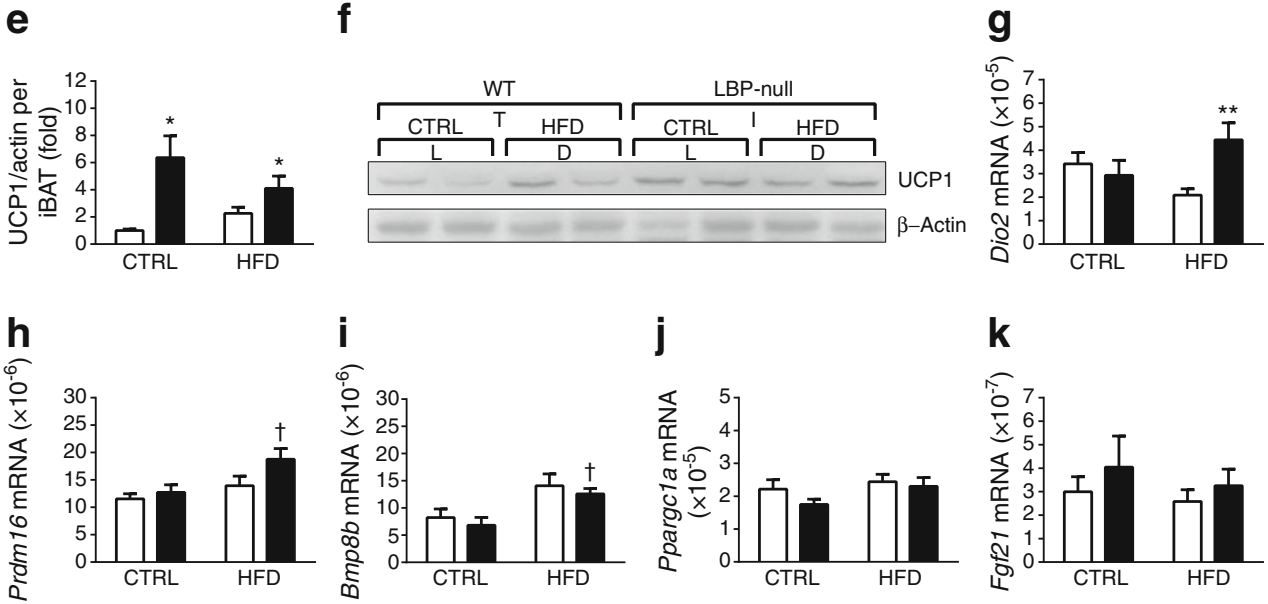

response to the lack of LBP. Consistent with this, the local temperature in the iBAT region was higher in $L b p$-null mice than in WT mice, at least under standard diet conditions (Fig. 5c).

Total UCP1 per iBAT depot was increased in $L b p$-null mice (Fig. 5e,f), although relative $U c p 1$ transcript levels were not significantly altered (Fig. 5d). This scenario is consistent with a long-term BAT recruitment process [23]. Transcript levels for other thermogenesis-related genes showed no major changes due to LBP ablation, except for a significant induction of Dio2 in the HFD group (Fig. $5 \mathrm{~g}-\mathrm{k}$ ).

Hepatic alterations in $\boldsymbol{L} \boldsymbol{b} \boldsymbol{p}$-null mice Liver size and hepatic triacylglycerol content were unaltered in $L b p$-null mice (data not shown). Expression of genes encoding gluconeogenesis-related enzymes (G6pase and Pepck) tended to be lower in Lbp-null mice (ESM Fig. 5a,b). The hepatic expression of Srebp, a master regulator of lipid synthesis, as well as Fas, Scdl and Acaca, was significantly reduced in Lbp-null mice under CTRL conditions (ESM Fig. 5c-f). Il6 gene expression was undetectable under CTRL conditions in both WT and $L b p$-null mice, but was strongly induced under HFD. This induction was impaired in $L b p$-null mice, a profile that paralleled circulating IL-6 levels (ESM Fig. 5i).

LBP expression negatively correlates with the expression of browning marker genes in human subcutaneous adipose tissue We analysed the expression of $L B P$ and browning marker genes in SAT and VAT biopsies from

morbidly obese patients. Anthropometrical and clinical variables included age $(48.3 \pm 9.1$ years $)$, BMI $\left(43.8 \pm 6.9 \mathrm{~kg} / \mathrm{m}^{2}\right)$, percentage fat mass $(56.1 \pm 10.1 \%)$, fasting glucose $(5.33 \pm 0.67 \mathrm{mmol} / \mathrm{l})$ and $M$ value $\left(23.5 \pm 13.3 \mu \mathrm{mol} \mathrm{kg} \mathrm{kg}^{-1} \mathrm{~min}^{-1}\right)$. We found significant negative correlations between $L B P$ transcript levels and the levels of transcripts for the browning marker genes UCP1, PRDM16, PPARGC1A and TMEM26 (the latter being a putative marker of beige/brite adipocytes [24]) in SAT (Table 1). No such correlation was found between $L B P$ expression and general markers of WAT such as PLIN1, LEP or DGAT1. In VAT, $L B P$ mRNA levels negatively correlated with TMEM26 mRNA levels and positively correlated with LEP mRNA levels (Table 1).

Table 1 Bivariate correlations between $\angle B P$ gene expression and the expression of browning marker genes in SAT and VAT from obese individuals $(n=38)$

\begin{tabular}{lll}
\hline Gene & SAT & VAT \\
\hline PRDM16 & $-0.32^{*}$ & -0.21 \\
PPARGC1A & $-0.51^{* *}$ & -0.28 \\
UCP1 & $-0.35^{*}$ & -0.08 \\
TMEM26 & $-0.33^{*}$ & $-0.49^{* *}$ \\
PLIN1 & -0.06 & -0.22 \\
LEP & 0.21 & $0.55^{* * *}$ \\
DGAT1 & 0.22 & 0.27 \\
\hline
\end{tabular}

Data are Spearman's $r$ coefficients ${ }^{*} p<0.05,{ }^{* *} p<0.01$ and ${ }^{* * *} p<0.001$ 


\section{Discussion}

In addition to its immunostimulatory functions [14], LBP has been identified as an adipokine associated with obesityinduced metabolic and proinflammatory disorders $[15,16]$. The promotion of BAT activity and, especially, of WAT browning are active research areas that hold promise for strategies aimed at protecting against obesity and associated metabolic abnormalities (e.g. hyperglycaemia and hyperlipidaemia) [24, 25]. Here, we report that both cold exposure and chronic treatment with a $\beta_{3}$-adrenergic agonist (two validated models for inducing WAT browning $[20,26]$ ) were associated with dramatic decreases in $L b p$ gene expression in iWAT. This phenomenon occurred in a cell-autonomous manner: $L b p$ expression was strongly repressed in cultures of adipocytes induced to acquire a beige/brite phenotype. Moreover, silencing of $L b p$ expression in adipocytes enhanced the expression of browning marker genes, whereas LBP treatment reversed this effect. As LBP is secreted by adipocytes, these data suggest that LBP may negatively regulate browning, apparently in an autocrine manner.

We observed that $L b p$-null mice were protected against body weight increases, which is consistent with the relationship observed between obesity and $L b p$ expression in the adipose tissues of human patients and mouse models [15]. In rodents, the thermogenic activity in BAT accounts for a significant proportion of whole body energy expenditure [27]; therefore, the observed activation of BAT in $L b p$-null mice may be involved in reducing weight gain. Moreover, although the estimated total thermogenic capacity of 'browned' WAT is much lower than that of BAT [23], browning of WAT is proposed to be relevant to protection against HFD-induced obesity $[8,9]$. Our results indicate that a lack of LBP in mice strongly enhances the browning of WAT, thus indicating that the in vitro function of LBP as negative regulator of browning also occurs in vivo. These findings support the concept that LBP, possibly acting in an autocrine manner, negatively regulates the browning process. The negative correlation between $L b p$ expression and marker genes of browning in human adipose samples is consistent with this scenario. The signs of BAT activation in mice lacking LBP appear to contrast with the minor association of BAT activation with $L b p$ downregulation in iBAT. It is possible that the effects of LBP deficiency on body weight in mice occur through alterations in $L b p$ expression in other tissues (e.g. nervous system) that indirectly contribute to regulation of BAT activity, and that the cell-autonomous effects of LBP deficiency are minor in the context of the whole animal. Furthermore, the lack of an overt increase in oxygen consumption (despite activated BAT and WAT browning) and unchanged food intake in $L b p$-null mice could be due to changes in these variables that are too small to be measurable but have long-term consequences on body weight. In any case, these observations warrant further research to determine whether impaired absorption of food might be different in $L b p$-null mice than in WT mice, given evidence for a role for LBP in gut biology [28]. In summary, the $L b p$-null model supports a role for LBP in the browning process, but the pleiotropic phenotype in these mice (see below) precludes definitive conclusions on the impact of browning induction on whole-body energy balance.

The obesity resistance, promotion of browning and increased BAT activity observed in $L b p$-null mice were accompanied by glucose intolerance and insulin insensitivity. Although seemingly unexpected, these findings can be viewed in relation to our current understanding that complex relationships exist between inflammatory pathways ( $L b p$-null mice also showed a decrease in systemic and local inflammatory markers, such as IL-6) and metabolic regulation. Numerous studies have demonstrated a positive association between serum LBP concentration and obesityassociated metabolic disturbances, including insulin resistance, in humans [29-33]. However, a discrepancy between circulating concentrations of innate immune system proteins in obese patients and the metabolic phenotype of the corresponding knockout mice has also been reported for lipocalin-2 [34, 35] and IL-6 [4, 36]. Other mouse models with loss of function of proinflammatory factors leading to impaired inflammation, such as the dominant-negative Tnfa transgenic mouse [37] and Fsp27-deficient mice [38], show reduced inflammation in adipose tissue associated with weight loss, but also insulin resistance. In fact, mice lacking LPS-binding capacity due to $C d 14$ knockout show reduced proinflammatory responses to LPS, but with significantly impaired glucose tolerance [39]. Thus, although current concepts tend to support the hypothesis that adipose tissue expansion in obesity is associated with local inflammation, which contributes to insulin resistance, recent research indicates that a certain degree of inflammatory response is essential for healthy adipose tissue expansion [37]. Our findings suggest the possibility that total impairment of the LPS signalling pathway limits an appropriate inflammatory response, abolishing the improvement in glucose tolerance that might be expected as a consequence of protection against obesity.

In conclusion, LBP appears to be a negative regulator of WAT browning and possibly of BAT activation. This action of LBP may be relevant to its role in favouring obesity and fat accretion. However, a total lack of LBP, even if protective against obesity, does not result in a systemic healthy phenotype. The complexities of homeostatic vs deleterious roles of inflammatory pathways in metabolic regulation are highlighted in the mouse model of $L b p$ gene ablation. Fine-tuning LBP levels should be considered a part of any prospective use of this factor in promoting healthy metabolism. 
Funding This work was partly supported by research grants from the Ministerio de Economía y Competitividad (PI11-00214, PI12/02631, SAF2014-55725 and BFU2015-70454-REDT) and FONDOS FEDER. CIBEROBN Fisiopatología de la Obesidad y Nutrición is an initiative of the Instituto de Salud Carlos III, Spain.

Duality of interest The authors declare that there is no duality of interest associated with this manuscript.

Contribution statement AG-N, TQ-L and MC performed experiments in rodent models; JMM-N and JMF-R performed the studies involving human tissue; AG-N, TQ-L, MG and JMM-N performed the cell culture experiments; all authors contributed to the study design and participated in data analysis; AG-N and FV wrote the manuscript; all authors contributed to drafting the manuscript or revised it critically for intellectual content; and all authors gave final approval of the version to be published. FV is the guarantor of this work

\section{References}

1. Erdmann J, Kallabis B, Oppel U, Sypchenko O, Wagenpfeil S, Schusdziarra V (2008) Development of hyperinsulinaemia and insulin resistance during the early stage of weight gain. Am J Physiol Endocrinol Metab 294:E568-E575

2. Saito M (2013) Brown adipose tissue as a regulator of energy expenditure and body fat in humans. Diabetes Metab J 37:22-29

3. Bartelt A, Bruns OT, Reimer R et al (2011) Brown adipose tissue activity controls triglyceride clearance. Nat Med 17:200-205

4. Stanford KI, Middelbeek RJ, Townsend KL et al (2013) Brown adipose tissue regulates glucose homeostasis and insulin sensitivity. J Clin Invest 123:215-223

5. Virtanen KA, Lidell ME, Orava J et al (2009) Functional brown adipose tissue in healthy adults. N Engl $\mathrm{J}$ Med 360:1518-1525

6. Cypess AM, White AP, Vernochet C et al (2013) Anatomical localization, gene expression profiling and functional characterization of adult human neck brown fat. Nat Med 19:635-639

7. Young P, Arch JR, Ashwell M (1984) Brown adipose tissue in the parametrial fat pad of the mouse. FEBS Lett 167:10-14

8. Guerra C, Koza RA, Yamashita H, Walsh K, Kozak LP (1998) Emergence of brown adipocytes in white fat in mice is under genetic control. Effects on body weight and adiposity. J Clin Invest 102:412-420

9. Seale P, Conroe HM, Estall J et al (2011) Prdm16 determines the thermogenic program of subcutaneous white adipose tissue in mice. J Clin Invest 121:96-105

10. Bartelt A, Heeren J (2014) Adipose tissue browning and metabolic health. Nat Rev Endocrinol 10:24-36

11. Nedergaard J, Cannon B (2014) The browning of white adipose tissue: some burning issues. Cell Metab 20:396-407

12. Grube BJ, Cochane CG, Ye RD et al (1994) Lipopolysaccharide binding protein expression in primary human hepatocytes and HepG2 hepatoma cells. J Biol Chem 269:8477-8482

13. Hailman E, Lichenstein HS, Wurfel MM et al (1994) Lipopolysaccharide (LPS)-binding protein accelerates the binding of LPS to CD14. J Exp Med 179:269-277

14. Tobias PS, Soldau K, Ulevitch RJ (1989) Identification of a lipid A binding site in the acute phase reactant lipopolysaccharide binding protein. J Biol Chem 264:10867-10871

15. Moreno-Navarrete JM, Escote X, Ortega F et al (2013) A role for adipocyte-derived lipopolysaccharide-binding protein in inflammation-and obesity-associated adipose tissue dysfunction. Diabetologia 56:2524-2537

16. Moreno-Navarrete JM, Escote X, Ortega F et al (2015) Lipopolysaccharide binding protein is an adipokine involved in the resilience of the mouse adipocyte to inflammation. Diabetologia 58:2424-2434

17. Aune UL, Ruiz L, Kajimura S (2013) Isolation and differentiation of stromal vascular cells to beige/brite cells. J Vis Exp 73:50191

18. Barbera MJ, Schluter A, Pedraza N, Iglesias R, Villarroya F, Giralt M (2001) Peroxisome proliferator-activated receptor alpha activates transcription of the Brown fat uncoupling protein-1 gene. A link between regulation of the thermogenic and lipid oxidation pathways in the brown fat cell. J Biol Chem 276: 1486-1493

19. Folch J, Lees M, Sloane Stanley GH (1957) A simple method for the isolation and purification of total lipids from animal tissues. J Biol Chem 226:497-509

20. Lee YH, Petkova AP, Mottillo EP, Granneman JG (2012) In vivo identification of bipotential adipocyte progenitors recruited by $\beta 3$ adrenoceptor activation and high-fat feeding. Cell Metab 15:480 491

21. Petrovic N, Walden TB, Shabalina IG, Timmons JA, Cannon B, Nedergaard J (2010) Chronic peroxisome proliferatoractivated receptor gamma (PPARgamma) activation of epididymally derived white adipocyte cultures reveals a population of thermogenically competent, UCP1-containing adipocytes molecularly distinct from classic brown adipocytes. J Biol Chem 285:7153-7164

22. Montgomery MK, Hallahan NL, Brown SH et al (2013) Mouse strain-dependent variation in obesity and glucose homeostasis in response to high-fat feeding. Diabetologia 56:1129-1139

23. Nedergaard J, Cannon B (1831) UCP1 mRNA does not produce heat. Biochim Biophys Acta 2013:943-949

24. Jespersen NZ, Larsen TJ, Peijs L et al (2013) A classical brown adipose tissue mRNA signature partly overlaps with brite in the supraclavicular region of adult humans. Cell Metab 17:798-805

25. Lichtenbelt W, Kingma B, van der Lans A, Schellen L (2014) Cold exposure-an approach to increasing energy expenditure in humans. Trends Endocrinol Metab 25:165-167

26. Lee P, Greenfield JR (2015) Non-pharmacological and pharmacological strategies of brown adipose tissue recruitment in humans. Mol Cell Endocrinol 418:184-190

27. Cannon B, Nedergaard J (2004) Brown adipose tissue: function and physiological significance. Physiol Rev 84:277-359

28. Vreugdenhil AC, Snoek AM, Greve JW, Buurman WA (2000) Lipopolysaccharide binding protein is vectorially secreted and transported by cultured intestinal epithelial cells and is present in the intestinal mucus of mice. J Immunol 165:4561-4566

29. Sun L, Yu Z, Ye X et al (2010) A marker of endotoxemia is associated with obesity and related metabolic disorders in apparently healthy Chinese. Diabetes Care 33:1925-1932

30. Moreno-Navarrete JM, Ortega F, Serino M et al (2012) Circulating lipopolysaccharide-binding protein (LBP) as a marker of obesityrelated insulin resistance. Int J Obes (Lond) 36:1442-1449

31. Serrano M, Moreno-Navarrete JM, Puig J et al (2013) Serum lipopolysaccharide-binding protein as a marker of atherosclerosis. Atherosclerosis 230:223-227

32. Gonzalez-Quintela A, Alonso M, Campos J, Vizcaino L, Loidi L, Gude F (2013) Determinants of serum concentrations of lipopolysaccharide-binding protein (LBP) in the adult population: the role of obesity. PLoS One 8:e54600

33. Liu X, Lu L, Yao P et al (2014) Lipopolysaccharide binding protein, obesity status and incidence of metabolic syndrome: a prospective study among middle-aged and older Chinese. Diabetologia 57: 1834-1841 
34. Moreno-Navarrete JM, Manco M, Ibanez J et al (2010) Metabolic endotoxemia and saturated fat contribute to circulating NGAL concentrations in subjects with insulin resistance. Int J Obes (Lond) 34 : 240-249

35. Guo H, Jin D, Zhang Y et al (2010) Lipocalin-2 deficiency impairs thermogenesis and potentiates diet-induced insulin resistance in mice. Diabetes 59:1376-1385

36. Matthews VB, Allen TL, Risis S et al (2010) Interleukin-6-deficient mice develop hepatic inflammation and systemic insulin resistance. Diabetologia 53:2431-2441
37. Wernstedt AI, Tao C, Morley TS et al (2014) Adipocyte inflammation is essential for healthy adipose tissue expansion and remodeling. Cell Metab 20:103-118

38. Zhou L, Park SY, Xu L et al (2015) Insulin resistance and white adipose tissue inflammation are uncoupled in energetically challenged Fsp27-deficient mice. Nat Commun 6:5949

39. Young JL, Mora A, Cerny A et al (2012) CD14 deficiency impacts glucose homeostasis in mice through altered adrenal tone. PLoS One 7:e29688 